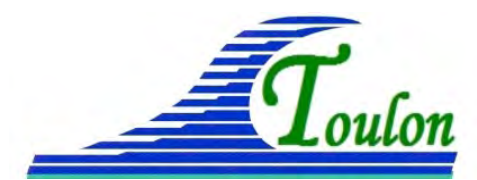
XIV èmes Journées Nationales Génie Côtier - Génie Civil
Toulon, 29 juin au $1^{\text {er }}$ juillet 2016

DOI:10.5150/jngcgc.2016.075 @ Editions Paralia CFL

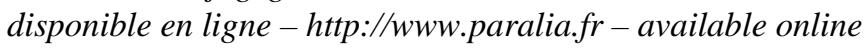

\title{
Etude comparative de l'érosion des falaises de Haouzia et de Sidi Bouzid (côte atlantique marocaine)
}

\author{
Soukaina KHOUAJA ${ }^{1}$, Mohamed OUADIA ${ }^{1}$, \\ M'hamed ABERKAN ${ }^{2}$, El Mehdi IRZAN ${ }^{1}$
}

\author{
1. Laboratoire Géosciences et Techniques de l'Environnement, Département de \\ Géologie, Faculté des Sciences. Route Ben Mâachou, BP 20, 24000 El Jadida, Maroc. \\ soukaina.khouaja@outlook.fr \\ 2. Université Mohammed V, Faculté des Sciences, Département des Sciences de la \\ Terre, BP 1014, Rabat, Maroc.
}

\section{Résumé :}

Ce travail, réalisé dans la région côtière marocaine entre Azemmour et Moulay Abdellah, intéresse la comparaison, pour la première fois, de l'érosion marine des deux falaises (Haouzia et Sidi Bouzid). Il montre que ces deux falaises et leurs extrémités (des deux côtés NE et $\mathrm{SW}$ ) ne se ressemblent pas et révèlent une érosion préférentielle et une variation spatiotemporelle remarquable.

A Haouzia, la falaise est vive, monocyclique moins haute et façonnée dans des calcarénites quaternaires moins indurées. A Sidi Bouzid, elle est polycyclique, vive, très haute et creusée, essentiellement, dans des calcaires marneux crétacés. Leur recul calculé est plus prononcé pour la falaise de Haouzia $(0,23 \mathrm{~m} / \mathrm{an})$ par rapport à celle de Sidi Bouzid (0,17 m/an).

Qualitativement, les valeurs importantes des paramètres des blocs à Sidi Bouzid s'expliquent par un héritage des caractéristiques des calcaires crétacés (lithologie, diaclases, plissement...) et leur faiblesse à Haouzia par la fragilité des calcarénites. Les valeurs des indices sont peu variables pour les deux falaises. Ls/E (longueur supérieur / épaisseur), élevé à Sidi Bouzid est dû aux diaclases espacées. La disposition des blocs est influencée par l'orientation des deux falaises, en plus de l'action d'un couloir au centre de Haouzia et de l'action de déferlement des vagues au Nord à Sidi Bouzid.

Quantitativement, les trois variables (nombre, densité et volume) sont plus prononcées à Sidi Bouzid du fait de l'impact de la fragilité des marnes, de l'espacement des diaclases, de la dynamique marine et de l'espace restreint du profil de plage.

Ces falaises sont différemment érodées suite à des facteurs variables et spécifiques pour chaque secteur. Cette érosion importante peut engendrer un risque difficilement contrôlable à long terme, pouvant endommager les infrastructures socio-économiques dans cette région si aucune mesure de protection n'est entreprise d'urgence.

Mots clés : Côte atlantique marocaine, Falaise, Haouzia, Sidi Bouzid, Erosion, Recul, Eboulis, Quantification, Dynamique marine, Evolution dans l'espaces, Evolution dans le temps, Risque. 


\section{Thème 7 - Risques côtiers}

\section{Introduction}

Ce travail a pour but d'essayer, pour la première fois, de quantifier et de comparer, qualitativement, l'érosion et les éboulements de deux falaises côtières (Haouzia et Sidi Bouzid) entre les belles plages renommées (Haouzia, El Jadida, Sidi Bouzid et Sidi El Abed). Il permet, également, de mettre en exergue les différents facteurs qui en sont la cause dont la dynamique marine et l'évolution dans l'espace et dans le temps ainsi que le risque que peut engendrer cette érosion et son impact sur les infrastructures avoisinantes et les activités socio-économiques.

\section{Situation géographique}

La région d'étude est située sur la côte atlantique marocaine, elle est limitée au NordEst, par la ville d'Azemmour et au Sud-Ouest, par la commune de Moulay Abdellah (figure 1). Les coordonnées Lambert des deux falaises étudiées, sont :

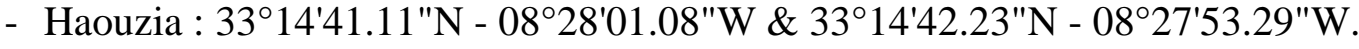

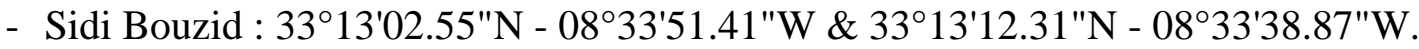

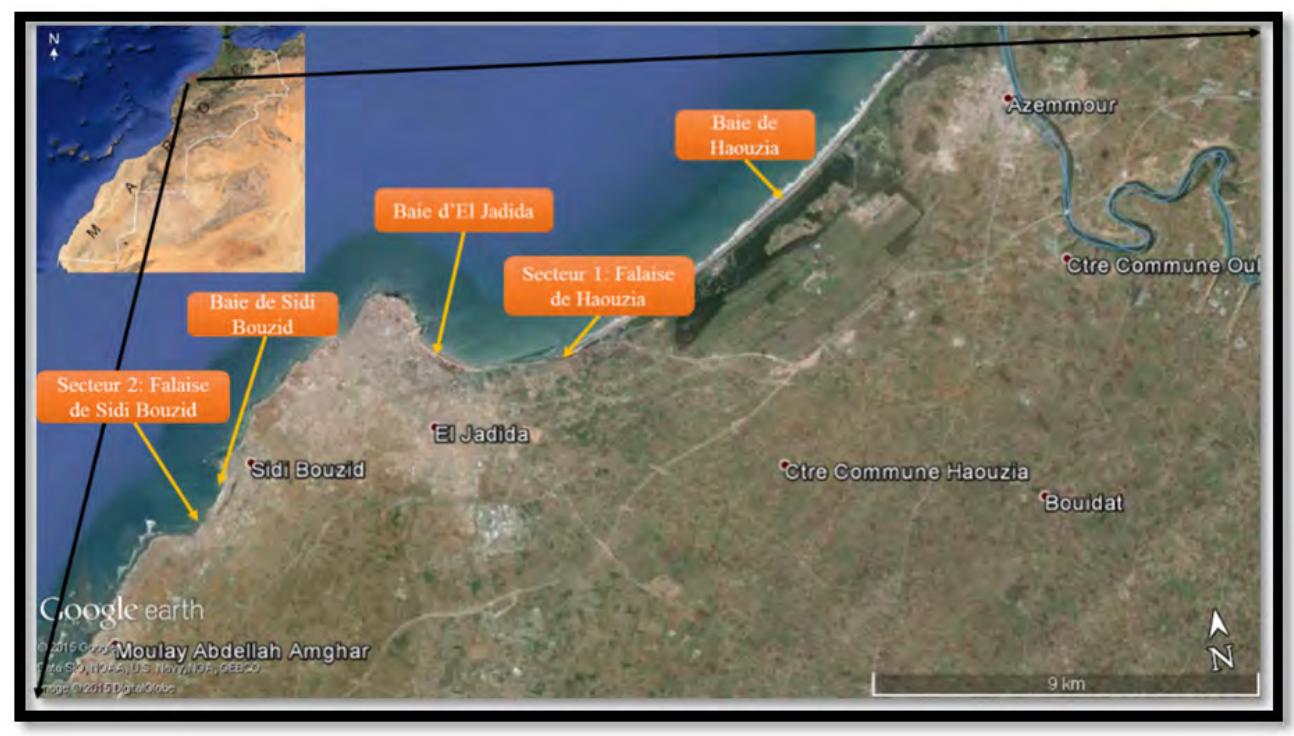

Figure 1. Situation géographique de la zone étudiée (photos Google Earth).

Les deux secteurs sont composés de falaises discontinues et interrompues par des petites plages sableuses. Le premier sépare deux baies : Haouzia au NE et El Jadida au SW. Le second sépare les baies de : Sidi Bouzid au NE et de Sidi El Abed au SW. Les deux falaises étudiées et dans ce travail, sont constituées, de calcarénites peu consolidées quaternaires dans le premier secteur et de calcaires marneux d’âge cénomanien (étudiés par GIGOUT, 1951 ; ABOUMARIA, 1993 ; OUADIA, 1998 et KHATMI, 1999), surmonté localement par des dépôts quaternaires à Sidi Bouzid. En plus de cette lithologie différentes dans les falaises, à hauteur, exposition, érosion et le volume des éboulis, résultant de leur éboulement sont contrastés. 


\section{XIV èmes Journées Nationales Génie Côtier - Génie Civil \\ Toulon, 29 juin au $1^{\text {er }}$ juillet 2016}

\section{Matériel et méthodes}

Dans ce travail, on a procédé à la mesure de plusieurs paramètres (définis ci-après) des blocs, en se basant sur la méthode proposée par (TRICART et al., 1955) légèrement modifiée pour adapter aux blocs. Les paramètres mesurés pour chaque bloc sont : Ls : longueur de la face supérieure ; Li : Longueur de la face inférieure; ls : largeur de la face supérieure; li : Largeur de la face inférieure; E : épaisseur et D : diagonale. La disposition des blocs, leur inclinaison et la direction de leur axe ainsi que leur inclinaison ont été mesurées. Après les mesures des paramètres, des indices (Longueur supérieur/longueur inférieur ; longueur supérieur/diagonale ; longueur supérieur/épaisseur) sont calculés et interprétés.

Pour l'étude de la falaise, la hauteur, la pente et les distances séparant leur pied du rivage, au niveau de la haute et de la basse marrée, ont été aussi mesurées.

Le matériel utilisé dans le terrain englobe essentiellement : mètre, boussole, GPS, niveleur, cartes topographiques et géologiques ainsi que des photographies aériennes et des photos Google Earth.

\section{Résultats et interprétation}

A partir de ces différentes analyses sur les falaises et les éboulements de la région apparait une grande différence entre les deux secteurs étudiés. Dans le premier secteur (Haouzia), la falaise est vive, monocyclique (attaquée par la mer pendant l'étage mellahien : Quaternaire final) et d'une hauteur qui peut atteindre 3 mètre. Ces falaises sont constituées de calcarénites quaternaires. Dans le second secteur de Sidi Bouzid, la falaise à une hauteur d'environ 10 mètre, elle est vive et polycyclique (en se basant sur la chronologie de divers stationnements marins quaternaires selon OUADIA (1998)) façonnée par au moins deux transgressions (ouljienne et mélahienne). Dans les deux extrémités (NE et $\mathrm{SW}$ ), les formations des falaises montrent de nombreux traits structuraux témoignant d'une activité tectonique ayant affecté cette région. Les memes constatation ont été faites par Ruellan et Auzende (1985) concernant la fracturation du plateau sous-marin d'El Jadida. La partie SW est constituée, essentiellement par des dépôts de pente quaternaires marins et continentaux déposés sur des calcaires marins crétacés. Quant à la partie NE, elle est composée d'alternances de bancs calcaires massifs et épais et de niveaux marneux. 

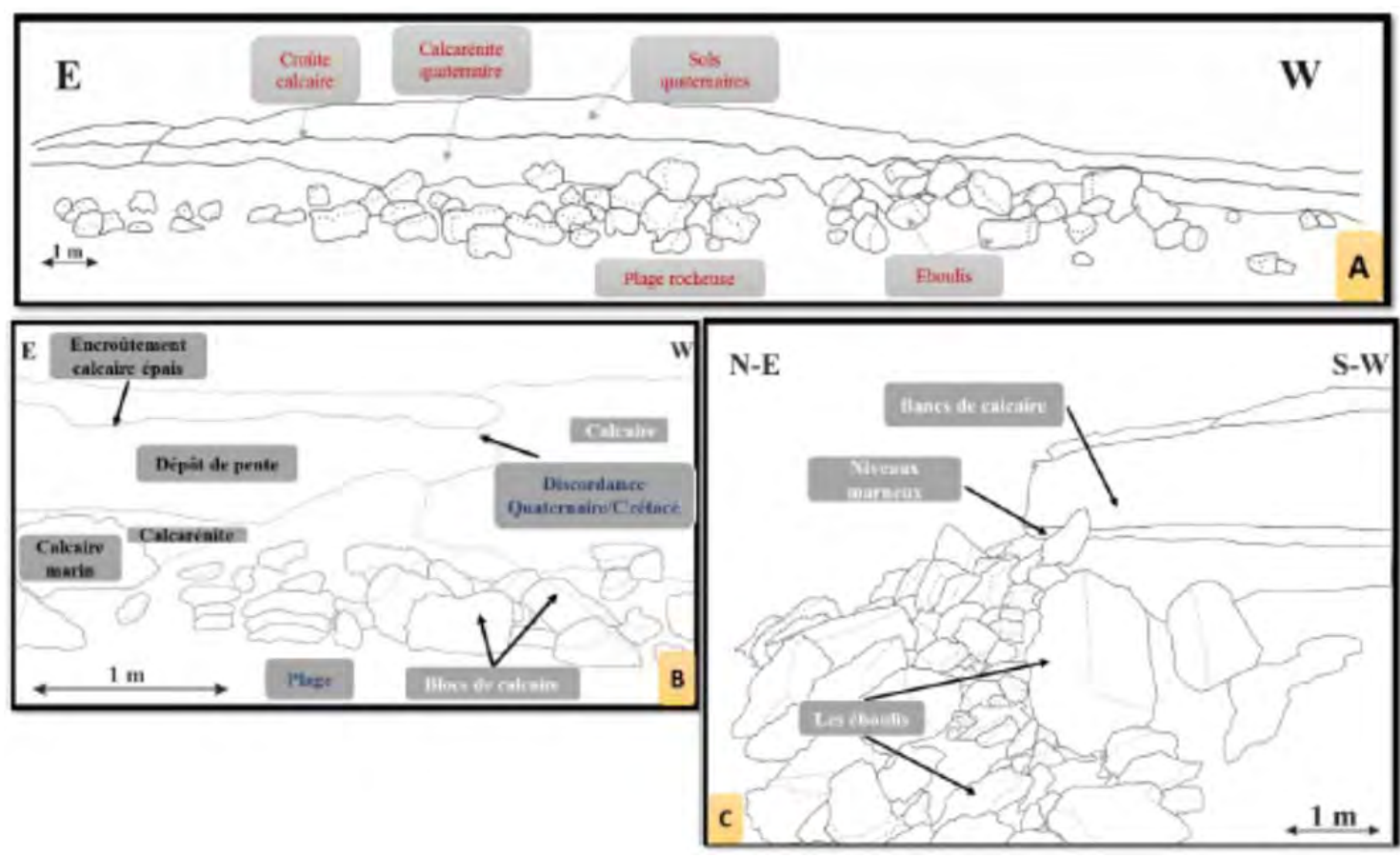

Figure 2. Lithologie des falaises étudiées

(A : Haouzia ; B : Sidi Bouzid Sud ; C : Sidi Bouzid Nord).

Dans les deux secteurs, la variation dans l'espace de l'érosion (en quantité et en qualité) de la falaise est, sans doute, en relation avec la diversité des facteurs l'ayant favorisée dont l'âge (durcissement avec le temps) et le degré de consolidation de ses formations mais également sa morphologie, son exposition à la dynamique marine et sa hauteur variable (figure 2).

La comparaison des photos Google Earth entre 2002 et 2015 et entre 2001 et 2015 pour Sidi Bouzid révèle un recul important à Haouzia $(23 \mathrm{~cm} / \mathrm{an})$ du fait de la fragilité des calcarénites (mentionnée par OUADIA et al., 2015) et l’âge récent (Quaternaire) de ses formations par rapport à Sidi Bouzid $(17 \mathrm{~cm} / \mathrm{an})$ sans oublier l'influence de la hauteur et de l'exposition de la falaise et d'autres facteurs qui ne sont pas les mêmes pour les deux secteurs (figure 3). La même constatation a été faite à Mohammedia (SBAI et al., 2004) et au Nord de Rabat (ABERKAN et al., 1997) qui présentent aussi des érosions différntielles.

Ces falaises, sont érodées, au sein du même secteur, en fonction de la nature et du degré des facteurs combinés (dynamique marin, morphologie et lithologie) qui sont variables dans l'espace.

Aussi, l'érosion de ces falaises présente un risque menaçant la stabilité de la région étudiée et ses infrastructures. 


\section{XIV vèmes Journées Nationales Génie Côtier - Génie Civil \\ Toulon, 29 juin au $1^{\text {er }}$ juillet 2016}

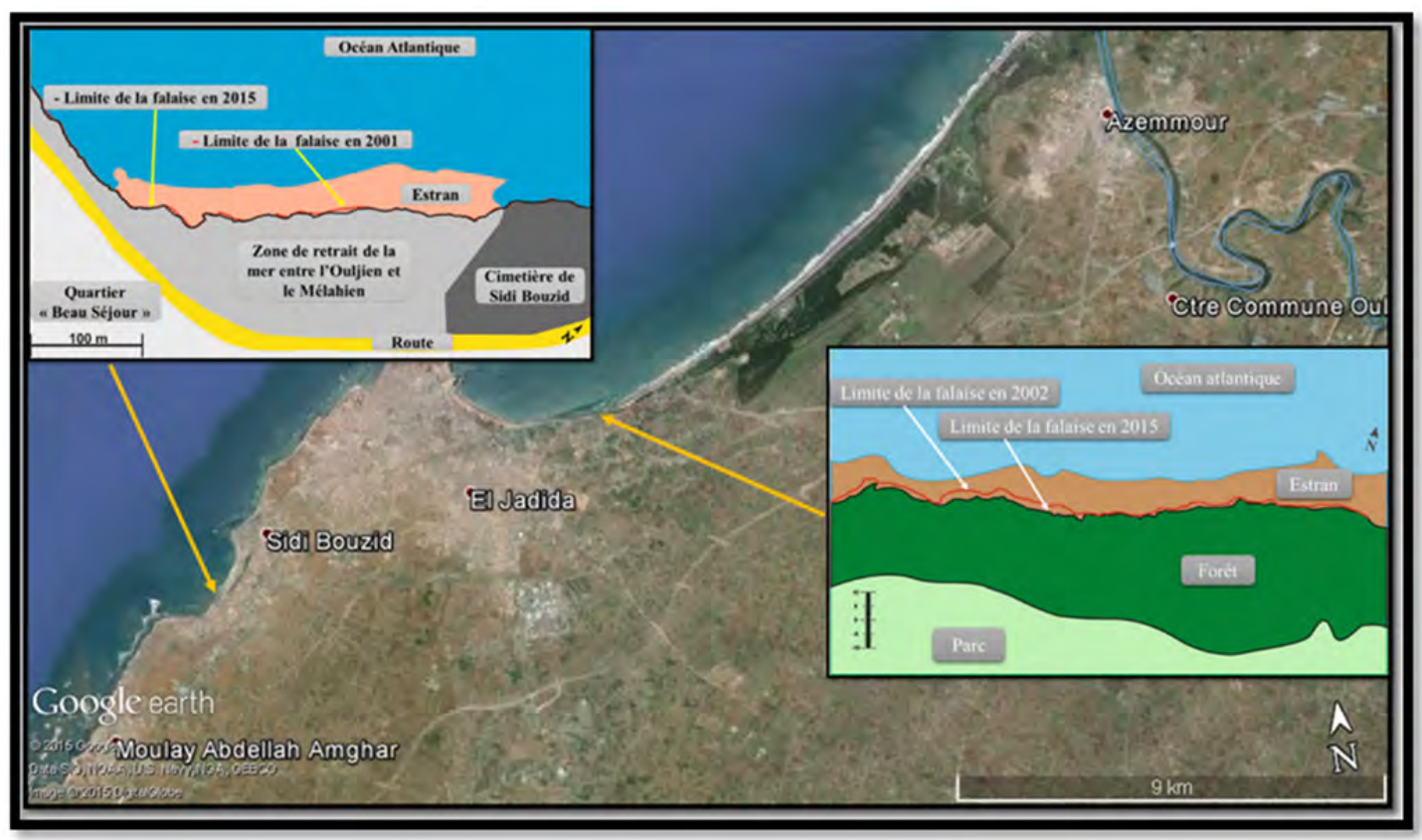

Figure 3. Evolution du recul des falaises de la région étudiée (photo Google Earth traitée).

Qualitativement (en relation avec la forme des blocs), les valeurs des paramètres des blocs sont plus importantes dans le secteur de Sidi Bouzid par rapport au celui de Haouzia (en particulier longueur supérieur et inferieur et diagonal). Dans ces deux secteurs, ces paramètres sont conditionnés par la lithologie (calcaires marneux et calcarénites moins résistants) des falaises et l'exposition de ces dernières. Mais à Sidi Bouzid, on note en plus que la longueur des blocs est conditionnée par l'espacement des diaclases, leur largeur par l'intercalation de bancs calcaires entre des niveaux marneux moins cohérants. Alors que l'épaisseur, quant à elle, est proportionnelle à celle des bancs massifs de calcaires qui est presque constante. La grande forme (en gros orthogonale) des blocs à Sidi Bouzid est essentiellement héritée sous l'influence de la fracturation des calcaires. Cependant à Haouzia, la nature, peu consolée des calcarénites peu âgées (quaternaires) permet aux blocs d'avoir une forme géométrique non particulière et relativement petite. Compte tenu de la force de l'érosion, la falaise de Haouzia est fragile et plus érodée que la falaise de Sidi Bouzid qui résiste, relativement, à cette érosion quoiqu'elle est plus haute (figure 4).

Les indices ( $\mathrm{Ls} / \mathrm{Li}$ et $\mathrm{Ls} / \mathrm{Li}$ ) des blocs, n’indiquent pas une grande différence entre les deux secteurs (Haouzia et Sidi Bouzid), mais, au sein de chaque secteur, une légère augmentation de leurs valeurs existe dans la partie SW à Haouzia et NE à Sidi Bouzid. Ce sont les parties de la falaise voisines du milieu littoral plus ou moins ouvertes et soumises aux vagues. Cependant, les valeurs élevées de Ls/E s’expliquent différemment 


\section{Thème 7 - Risques côtiers}

dans les deux secteurs. A Haouzia c'est à cause de l'épaisseur faible des blocs, en partie héritée, mais aussi réduites postérieurement à leur mise en place (Fig.5).

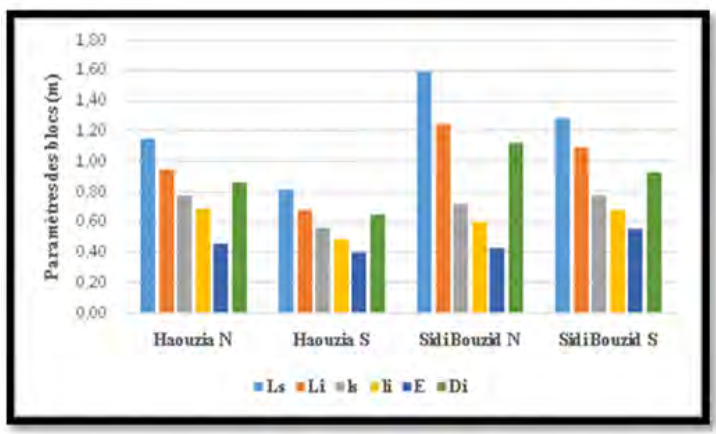

Figure 4. Evolution des paramètres des blocs (Ls ; Li ; ls ;li ; E ; Di).

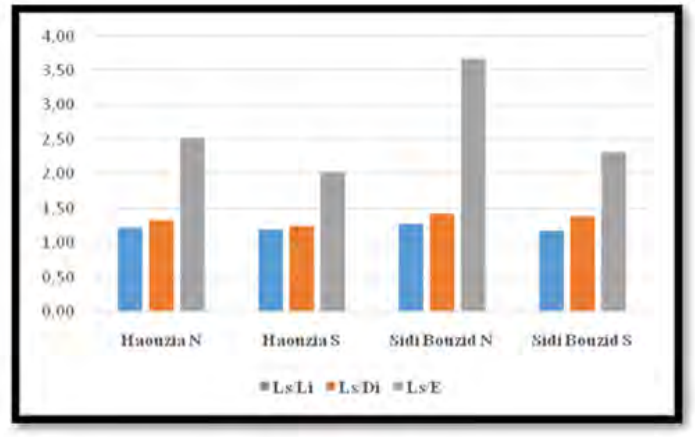

Figure 5. Evolution des indices des blocs (Ls/Li; Ls/Di ; Ls/E).

Concernant la disposition des blocs, les valeurs de la direction des axes des blocs, de l'inclinaison des blocs et de la direction de l'inclinaison des blocs évoluent de la même, manière dans les deux secteurs. Elles sont relativement plus élevées à Haouzia Sud et à Sidi Bouzid Nord suite à l'influence d'un couloir sur la plage pour la partie Sud de Haouzia et le déferlement des vagues pour la partie Nord de Sidi Bouzid (figure 6).

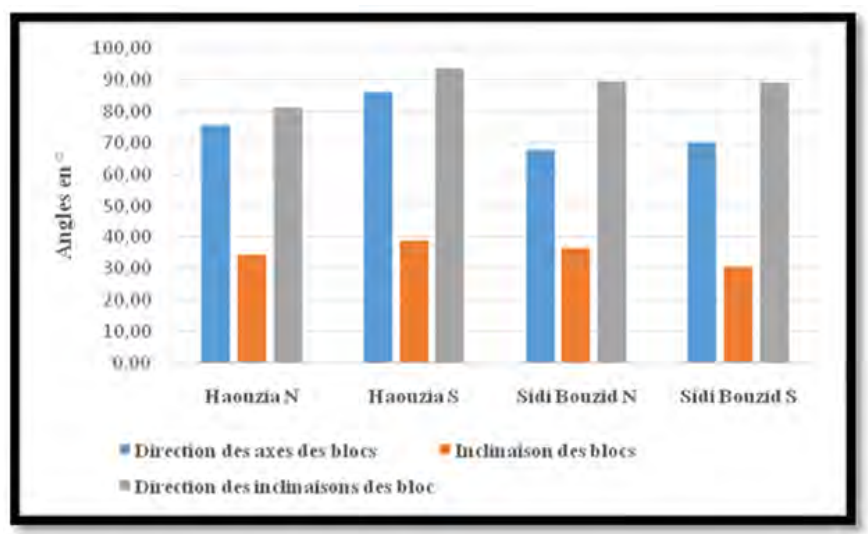

Figure 6. Evolution de la direction de l'inclinaison et de l'axe des blocs et de leurs inclinaisons.

L'analyse des blocs issus de l'érosion des falaises étudiées révèle que, le nombre, la densité et le volume sont différents d'un secteur à l'autre. Ils sont plus importants à Sidi Bouzid plus qu'à Haouzia à cause de la hauteur et de l'exposition importante de la falaise et de la lithologie des formations (alternances de calcaires et de marnes) ainsi que de la faible surface d'accumulation des blocs au pied de la falaise sans oublier l'impact des diaclases et des plissements fréquents à Sidi Bouzid. A Haouzia, c'est le contraire sauf que l’âge quaternaire des calcarénites rend celles-ci moins résistantes. 


\section{XIV èmes Journées Nationales Génie Côtier - Génie Civil \\ Toulon, 29 juin au $1^{\text {er }}$ juillet 2016}

Cela est expliqué par la lithologie (calcaire crétacé et calcarénite encroûtée) et la présence des diaclases plus ou moins espacées (blocs denses et volumineux) ce qui rend la fragmentation, après leur mise en place, des blocs très difficile. Contrairement à la falaise de Haouzia, pour la falaise de Sidi Bouzid, sa nature lithologique et la présence d'une deuxième falaise au pied de F1 (partie Sud) ont joué le rôle le plus important dans la fragmentation des blocs (figures 7 et 8).

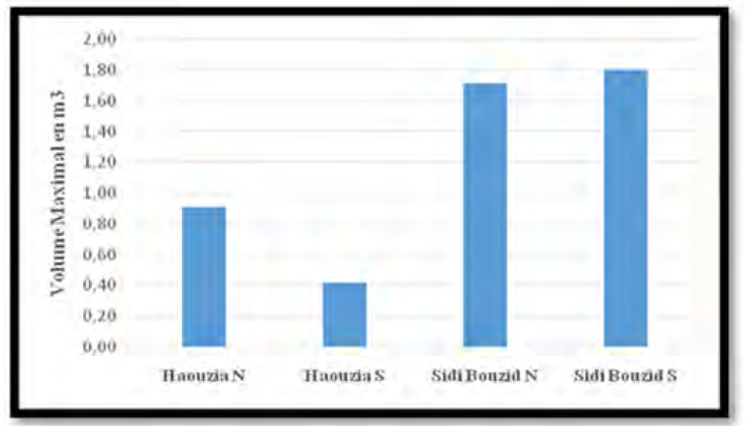

Figure 7. Evolution du volume maximal des blocs.

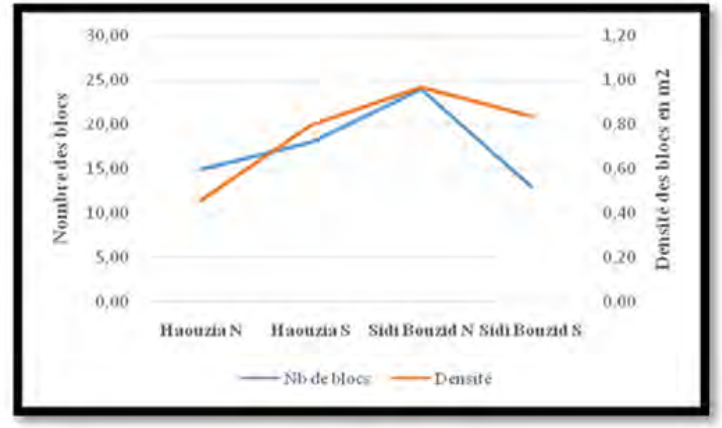

Figure 8. Evolution du nombre et de la densité des blocs.

\section{Conclusion}

Ce travail, montre que les falaises comparées de Haouzia et de Sidi Bouzidsont très différentes l'une de l'autre. Leurs hauteur, exposition, étendue ainsi que formations (nature, âge et tectonique) ne se ressemblent pas.

Le premier secteur (Haouzia), sa falaise vive et monocyclique est façonnée dans des calcarénites quaternaire, de hauteur d'environ $3 \mathrm{~m}$, associée à deux autres petites falaises (F2 et F3 visibles lors des basses marrées). Le deuxième secteur (Sidi Bouzid), sa falaise vive et polycyclique est constituée d'une alternance de bancs des calcaires crétacés avec des niveaux de marnes au Nord, façonnée dans des dépôts quaternaires superposant des calcaires crétacés au Sud. Cette falaise est très haute par rapport à la falaise de Haouzia, de $10 \mathrm{~m}$ de hauteur et associée à une petite falaise F2 qui affleure dans la partie Nord au moment de fortes tempêtes.

Quantitativement, le nombre, les valeurs de la densité et du volume sont plus importants dans le secteur de Sidi Bouzid par rapport à celui de Haouzia. Cela est expliqué par l'influence de la nature lithologique des formations, la hauteur, le type de profil et la présence des diaclases. Tous ces facteurs jouent sur le degré d'érosion et l'instabilité des falaises. Pour les indices de blocs, le seul indice qui fait la différence entre les deux secteurs c'est l'indice Ls/ E, qui est très élevé dans le secteur Sidi Bouzid. Cela est dû à la longueur la plus importante des blocs dans ce secteur (diaclases plus espacées).

Les valeurs des paramètres sont élevées dans à Sidi Bouzid. Elles sont conditionnées par lithologique des formations, la forme héritée des blocs, de l'espacement entre les diaclases et aussi la nature de profil (sableux ou rocheux/ restreint ou long). 


\section{Thème 7 - Risques côtiers}

On peut conclure que le phénomène d'érosion est conditionné par plusieurs facteurs particuliers à chaque partie de la région étudiée. Le secteur Haouzia est plus touché par l'érosion que le secteur Sidi Bouzid, cela est témoigné par l'ampleur du recul de la falaise qui est plus importante à Haouzia (0,23 m/an) par rapport à Sidi Bouzid $(0,17 \mathrm{~m} / \mathrm{an})$.

En conclusion, il parait nettement que l'érosion dans la région d'étude présente un vrai risque qui menace la stabilité de cette dernière plus les infrastructures installées et avoisinant ces falaises objet de ce travail.

\section{Références}

ABERKAN M., ADIL S., ZANNIBI F. (1997). Le recul de la falaise côtière et le problème d'aménagement le long de la côte atlantique marocaine. $2^{\text {ème }}$ Rencontre de l'Hydrodynamique marine. Ben M'SIK Casablanca.

ABOUMARIA K. (1993). Les formations quaternaires du Sahel des Doukkala (Méséta occidentale marocaine) : mise en place et évolution post-sédimentaire. Thèse de doctorat $3^{\text {éme }}$ cycle, Fac. Sci, Rabat, 186 p.

GIGOUT M. (1951). Etude géologique sur la Méséta marocaine occidentale (arrièrepays de Casablanca, Mazagan et Safi). Trav. Inst. Sc. Chérifien, 3, et Not. Mém. Serv. Géol. Maroc, n 86, 2 t., 507 p.

KHATMI S. (1999). La couverture méso-cénozoïque des Doukkala (Meséta côtière, Maroc) : Stratigraphie et évolution géodynamique. Thèse de doctorat $3^{\text {éme }}$ cycle, El Jadida, 124 p.

OUADIA M. (1998). Les formations plioquaternaires dans le domaine mésétien occidental entre Casablanca et Safi : Géomorphologie, Sédimentologie, Paléoenvironnements quaternaires et Evolution actuelle. Thèse doctorat d'état, Univ. Mohammed V, Rabat, 319 p.

OUADIA M., ABERKAN M., ABOUMARIA K. (2015). Le complexe dunaire quaternaire du JorfLasfar (Meseta côtière occidentale marocaine) : lithostratigraphie, sédimentologie et néotectonique. Revue Paralia, Vol. 8, $\mathrm{n}^{\circ} 2.1-\mathrm{n}^{\circ} 2.14$. http://dx.doi.org/10.5150/revue-paralia.2015.n02

REULLAN E., AUZENDE J.M. (1985) - Structure et évolution du plateau sous-marin de El Jadida (Mazagan, Ouest Maroc). Bull. Soc. géol. France, (8), t. I, n 1, p. 103114.

SBAI F., LABRAIMI M., HADDANE M. (2004). Evaluation du recul du trait de côte sur une portion du littoral atlantique marocain de Mohammedia. PANGEA 41/42, juindécembre.

TRICART J., JOLY F., RAYNAL R. (1955). Etude morphométrique des galets nordafricains. Notes Serv. Géol. Maroc, t. 13, n 128, pp 49-83. 